$\mathrm{J}$ o u r n a l of

Mathematics

and Applications

No 35, pp 5-21 (2012)

\title{
The Fourier method in three-dimensional dynamical problems of the hemitropic theory of elasticity
}

\author{
Yuri Bezhuashvili and Roland Rukhadze
}

Submitted by: Jan Stankiewicz

\begin{abstract}
The basic three-dimensional dynamical problems for a hemitropic (non-centrally symmetric) micropolar elastic medium are considered. Using the Fourier method, the solvability of the formulated by us problems is proved in a classical sense.
\end{abstract}

AMS Subject Classification:

Key Words and Phrases:

\section{Basic Notation and Equations}

Let $D \subset R^{3}$ be a finite domain bounded by a closed surface $S$ of the class $C^{2, \alpha}$; $0<\alpha \leq 1 ; \bar{D}=D \cup S, L=(0, e), \bar{L}=[0, e], \Omega=D \times L$ be a cylinder in $R^{4}$, $\bar{\Omega}=\bar{D} \times \bar{L}$.

A system of differential equations of dynamics for the hemitropic micropolar elastic medium is of the form $[1,2]$ :

$$
\left\{\begin{array}{l}
(\mu+\alpha) \Delta u+(\lambda+\mu-\alpha) \operatorname{grad} \operatorname{div} u+(v+\eta) \Delta \omega \\
\quad+(\delta+v-\eta) \operatorname{grad} \operatorname{div} \omega+2 \alpha \operatorname{rot} \omega+X(x, t)=\rho \frac{\partial^{2} u}{\partial t^{2}} \\
(\nu+\beta) \Delta \omega-4 \alpha \omega+(\varepsilon+\nu-\beta) \operatorname{grad} \operatorname{div} \omega+(v+\eta) \Delta u \\
\quad+(\delta+v-\eta) \operatorname{grad} \operatorname{div} u+2 \alpha \operatorname{rot} u+4 \eta \operatorname{rot} \omega \\
\quad+Y(x, t)=\mathcal{J} \frac{\partial^{2} \omega}{\partial t^{2}}
\end{array}\right.
$$

where $\Delta$ is the three-dimensional Laplace operator; $u(x, t)=$ $\left(u_{1}, u_{2}, u_{3}\right)$ is the displacement vector; $\omega(x, t)=\left(\omega_{1}, \omega_{2}, \omega_{3}\right)$ is the vector of rotation; $x=\left(x_{1}, x_{2}, x_{3}\right)$ is a point in $R^{3} ; t$ is time; $X(x, t)$ is the vector of body forces;

COPYRIGHT (C) by Publishing Department Rzeszów University of Technology P.O. Box 85, 35-959 Rzeszów, Poland 
$Y(x, t)$ is the volumetrical moment; $\rho$ is the medium density; $\mathcal{J}$ is a moment of inertia, and $\lambda, \mu, \alpha, \varepsilon, \nu, \beta, v, \eta, \delta$ are the known elastic constants satisfying the following conditions: $\mu>0, \alpha>0,3 \lambda+2 \mu>0, \mu \nu-v^{2}>0, \alpha \beta-\eta^{2}>0$, $(3 \lambda+2 \mu)(3 \varepsilon+2 \nu)-(3 \delta+2 v)^{2}>0$.

For the sake of brevity, the basic equations will be written in a matrix form. Towards this end, we adopt the following agreement: if we multiply the matrix $A=$ $\left\|a_{i j}\right\|_{m \times n}$ of dimension $n$ by the $u=\left(u_{1}, u_{2}, \ldots, u_{n}\right)$-dimensional vector $n$, then the vector is assumed to be a one-column matrix $u=\left\|u_{i}\right\|_{n \times 1}$, and the product $A u$ is an $m$-dimensional vector.

The system (1) can be represented in a vector-matrix form as follows:

$$
M(\partial x) V(x, t)+F(x, t)=r \frac{\partial^{2} V}{\partial t^{2}},
$$

where $M(\partial x)=\left\|M_{k j}(\partial x)\right\|_{6 \times 6}$, and also

$$
\begin{aligned}
M_{k j}(\partial x)= & (\mu+\alpha) \delta_{k j} \Delta+(\lambda+\mu-\alpha) \frac{\partial^{2}}{\partial x_{k} \partial x_{j}} \quad \text { for } \quad k, j=1,2,3, \\
M_{k j}(\partial x)= & (v+\eta) \delta_{k j} \Delta+(\delta+v-\eta) \frac{\partial^{2}}{\partial x_{k} \partial x_{j}}-2 \alpha \sum_{e=1}^{3} \varepsilon_{k j} \frac{\partial}{\partial x_{e}} \\
\text { for } k=1,2,3, \quad j=4,5,6 \text { and } k=4,5,6, \quad j=1,2,3 & \\
M_{k j}(\partial x)= & ((\nu+\beta) \Delta-4 \alpha) \delta_{k j}+(\varepsilon+\nu-\beta) \frac{\partial^{2}}{\partial x_{k} \partial x_{j}} \\
& -4 \eta \sum_{e=1}^{3} \varepsilon_{k j e} \frac{\partial}{\partial x_{e}} \quad \text { for } \quad k, j=4,5,6
\end{aligned}
$$

$\delta_{k j}$ is the Kronecker symbol, $\varepsilon_{k} j e$ is the Levy-Civita symbol,

$$
\begin{gathered}
F(x, t)=(X(x, t), Y(x, t))=\left(F_{1}, F_{2}, \ldots, F_{6}\right) ; \\
V(x, t)=(u(x, t), \omega(x, t))=\left(u_{1}, u_{2}, u_{3}, \omega_{1}, \omega_{2}, \omega_{3}\right)=\left(v_{1}, v_{2}, \ldots, v_{6}\right) ;
\end{gathered}
$$

$r$ is the diagonal matrix of dimension $6 \times 6 ; r=\left\|r_{k j}\right\|_{6 \times 6}, r_{k j}=0$, where $k \neq j$, $r_{i i}=\rho$ for $i=1,2,3 ; r_{i i}=\mathcal{J}$ for $i=4,5,6$.

The force stress $r^{(n)}(x, t)=\left(r_{1}^{(n)}(x, t), r_{2}^{(n)}(x, t), r_{3}^{(n)}(x, t)\right)$ and the moment stress $\mu^{(n))}(x, t)=\left(\mu_{1}^{(n)}(x, t), \mu_{2}^{(n)}(x, t), \mu_{3}^{(n)}(x, t)\right)$ at the point $x$ and time $t$ directed to $n=\left(n_{1}, n_{2}, n_{3}\right)$ are defined by the formulas $[1,2]$ :

$$
\begin{aligned}
r_{i}^{(n)} & =\lambda n_{i} \operatorname{div} u+(\mu+\alpha) \sum_{j=1}^{3} \frac{\partial u_{i}}{\partial x_{j}} n_{j}+(\mu-\alpha) \sum_{j=1}^{3} \frac{\partial u_{j}}{\partial x_{i}} n_{j}+ \\
& +\delta n_{i} \operatorname{div} \omega+(v+\eta) \sum_{j=1}^{3} \frac{\partial \omega_{i}}{\partial x_{j}} n_{j}+(v-\eta) \sum_{j=1}^{3} \frac{\partial \omega_{j}}{\partial x_{i}} n_{j}
\end{aligned}
$$


The Fourier method in three-dimensional dynamical ...

$$
\begin{gathered}
+2 \alpha \sum_{k, j=1}^{3} \varepsilon_{i j k} \omega_{k} n_{j}, \quad i=1,2,3 \\
\mu_{i}^{(n)}=\delta n_{i} \operatorname{div} u+(v+\eta) \sum_{j=1}^{3} \frac{\partial u_{i}}{\partial x_{j}} n_{j}+(v-\eta) \sum_{j=1}^{3} \frac{\partial u_{j}}{\partial x_{i}} n_{j} \\
+\varepsilon n_{i} \operatorname{div} \omega+(\nu+\beta) \sum_{j=1}^{3} \frac{\partial \omega_{i}}{\partial x_{j}} n_{j}+(\nu-\beta) \sum_{j=1}^{3} \frac{\partial \omega_{j}}{\partial x_{i}} n_{j} \\
+(v+\eta) \sum_{k, j=1}^{3} \varepsilon_{j k i} \omega_{k} n_{j}+(v-\eta) \sum_{k, j=1}^{3} \varepsilon_{i j k} \omega_{k} n_{j}, \quad i=1,2,3 .
\end{gathered}
$$

We introduce a matrix differential operator of dimension $6 \times 6$ :

$$
\begin{aligned}
T( & \partial x, n(x))=\left\|T_{k j}(\partial x, n(x))\right\|_{6 \times 6}, \\
T_{k j}(\partial x, n(x))= & (\mu+\alpha) \delta_{k j} \frac{\partial}{\partial n(x)}+(\mu-\alpha) n_{j} \frac{\partial}{\partial x_{k}} \\
& +\lambda n_{k} \frac{\partial}{\partial x_{j}}, \quad k, j=1,2,3 ; \\
T_{k j}(\partial x, n(x))= & (v+\eta) \delta_{k j} \frac{\partial}{\partial n(x)}+(v-\eta) n_{j} \frac{\partial}{\partial x_{k}}+\delta n_{k} \frac{\partial}{\partial x_{j}} \\
& -2 \alpha \sum_{e=1}^{3} \varepsilon_{k j e} n_{e}, \quad k=1,2,3, \quad j=4,5,6 ; \\
T_{k j}(\partial x, n(x))= & (v+\eta) \delta_{k j} \frac{\partial}{\partial n(x)}+(v-\eta) n_{j} \frac{\partial}{\partial x_{k}} \\
& +\delta n_{k} \frac{\partial}{\partial x_{j}}, \quad k=4,5,6, \quad j=1,2,3 ; \\
T_{k j}(\partial x, n(x))= & (\nu+\beta) \delta_{k j} \frac{\partial}{\partial n(x)}+(\nu-\beta) n_{j} \frac{\partial}{\partial x_{k}}+\beta n_{k} \frac{\partial}{\partial x_{j}} \\
& -2 \nu \sum_{e=1}^{3} \varepsilon_{k j e} n_{e}, \quad k, j=4,5,6 .
\end{aligned}
$$

$T(\partial x, n(x))$ will be called a stress operator of the hemitropic theory of elasticity.

It is not difficult to check that

$$
T(\partial x, n(x)) V=\left(r^{(n)}(V), \mu^{(n)}(V)\right) .
$$

Note that $n(x)$ is an arbitrary unit vector at the point $x$. If $x \in S$, then $n(x)$ is the unit vector of the outer normal with respect to $D$. 


\section{Statement of the Basic Problems. The Condi- tions for the Given Vector-Functions}

Let the hemitropic homogeneous elastic medium occupy the domain $D$. We consider the following two basic problems: find in a cylinder $\Omega$ a regular vector $V(x, t)$ $\left(x \in D, t \in L, V_{i} \in C^{1}(\bar{\Omega}) \cap C^{2}(\Omega), i=\overline{1,6}\right)$ satisfying

1) the equation

$$
\forall(x, t) \in \Omega: M(\partial x) V(x, t)-r \frac{\partial^{2} V(x, t)}{\partial t^{2}}=-F(x, t) ;
$$

2) the initial conditions

$$
\forall x \in \bar{D}: \lim _{t \rightarrow 0+} V(x, t)=\varphi(x), \quad \lim _{t \rightarrow 0+} \frac{\partial V(x, t)}{\partial t}=\psi(x)
$$

3) one of the boundary conditions

$$
\begin{aligned}
& \forall(z, t) \in S \times \bar{L}: \lim _{D \ni x \rightarrow z \in S} V(x, t)=0, \text { for the first problem, } \\
& \forall(z, t) \in S \times \bar{L}: \lim _{D \ni x \rightarrow z \in S} T(\partial x, n(x)) V(x, t)=0
\end{aligned}
$$

for the second problem.

The first problem we denote by $(I)_{F, \varphi, \psi}$, and the second one by $(I I)_{F, \varphi, \psi}$.

The given vector-functions $F, \varphi, \psi$ are assumed to satisfy the following conditions:

1) $F(\cdot, \cdot) \in C^{2}(\bar{\Omega})$, and the third order derivatives belong to the class $L_{2}(D)$. Moreover,

$$
\left.F\right|_{S}=\left.M F\right|_{S}=0, \quad t \in \bar{L} \quad \text { for the problem }(I)_{F, \varphi, \psi}
$$

and

$$
\left.T F\right|_{S}=0, \quad t \in \bar{L} \quad \text { for the problem }(I I)_{F, \varphi, \psi}
$$

2) $\varphi \in C^{3}(\bar{D})$, and the fourth order derivatives belong to the class $L_{2}(D)$. Moreover,

$$
\left.\varphi\right|_{S}=\left.M \varphi\right|_{S}=0, \quad \text { for the problem }(I)_{F, \varphi, \psi}
$$

and

$$
\left.T \varphi\right|_{S}=\left.T M \varphi\right|_{S}=0, \quad \text { for the problem }(I I)_{F, \varphi, \psi} ;
$$

3) $\psi \in C^{2}(\bar{D})$, and the third order derivatives belong to the class $L_{2}(D)$. Moreover,

$$
\left.\psi\right|_{S}=\left.M \psi\right|_{S}=0, \quad \text { for the problem }(I)_{F, \varphi, \psi}
$$

and

$$
\left.T \psi\right|_{S}=0, \quad \text { for the problem }(I I)_{F, \varphi, \psi} .
$$

The symbol $\left.\cdot\right|_{S}$ denotes the narrowing to $K S$. 
The Fourier method in three-dimensional dynamical ...

\section{Green's Formulas. The Uniqueness of Regular Solutions of the Problems Formulated Above}

Let $V(x)=(u, \omega)$ and $V^{\prime}(x)=\left(u^{\prime}, \omega^{\prime}\right)$ be arbitrary six-component vectors of the class $C^{1}(\bar{D})$ whose second derivatives belong to the class $L_{2}(D)$. Then the following Green's formulas are valid [3]:

$$
\begin{gathered}
\int_{D}\left(V^{\prime} M(\partial x) V+E\left(V^{\prime}, V\right)\right) d x=\int_{S} V^{\prime} T V d S \\
\int_{D}\left(V^{\prime} M(\partial x) V-V M(\partial x) V^{\prime}\right) d x=\int_{S}\left(V^{\prime} T V-V T V^{\prime}\right) d S
\end{gathered}
$$

where

$$
\begin{aligned}
E\left(V^{\prime}, V\right)= & \sum_{k, j=1}^{3}\left((\mu+\alpha) u_{k j}^{\prime} u_{k j}+(\mu-\alpha) u_{k j}^{\prime} u_{j k}\right. \\
& +(v+\eta) u_{k j}^{\prime} \omega_{k j}+(v-\eta) u_{k j}^{\prime} \omega_{j k}+(\nu+\beta) \omega_{k j}^{\prime} \omega_{k j} \\
& +(\nu-\beta) \omega_{k j}^{\prime} \omega_{j k}+(v+\eta) \omega_{k j}^{\prime} u_{k j}+(v-\eta) \omega_{k j}^{\prime} u_{j k} \\
& \left.+\delta\left(u_{k k}^{\prime} \omega_{j j}+u_{k k} \omega_{j j}^{\prime}\right)+\lambda u_{k k}^{\prime} u_{j j}+\varepsilon \omega_{k k}^{\prime} \omega_{j j}\right)
\end{aligned}
$$

where $u_{k j}=\frac{\partial u_{j}}{\partial x_{k}}-\sum_{e=1}^{3} \varepsilon_{k j e} \omega_{e}, \omega_{k j}=\frac{\partial \omega_{j}}{\partial x_{k}}$. From (3) we have

$$
\begin{gathered}
E(V, V)=\frac{3 \lambda+2 \mu}{3}\left(\operatorname{div} u+\frac{3 \delta+2 v}{2 \lambda+2 \mu} \operatorname{div} \omega\right)^{2}+ \\
+\frac{1}{3}\left(3 \varepsilon+2 \nu-\frac{(3 \delta+2 v)^{2}}{3 \lambda+2 \mu}\right)(\operatorname{div} \omega)^{2}+ \\
+\frac{\mu}{2} \sum_{k \neq j=1}\left(\frac{\partial u_{k}}{\partial x_{j}}+\frac{\partial u_{j}}{\partial x_{k}}+\frac{v}{\mu}\left(\frac{\partial \omega_{k}}{\partial x_{j}}+\frac{\partial \omega_{j}}{\partial x_{k}}\right)\right)^{2}+ \\
+\frac{\mu}{3} \sum_{k, j=1}^{3}\left(\frac{\partial u_{k}}{\partial x_{k}}-\frac{\partial u_{j}}{\partial x_{j}}+\frac{v}{\mu}\left(\frac{\partial \omega_{k}}{\partial x_{k}}-\frac{\partial \omega_{j}}{\partial x_{j}}\right)\right)^{2}+ \\
+\left(\nu-\frac{v^{2}}{\mu}\right)\left(\frac{1}{2} \sum_{k \neq j=1}\left(\frac{\partial \omega_{k}}{\partial x_{j}}+\frac{\partial \omega_{j}}{\partial x_{k}}\right)^{2}+\frac{1}{3} \sum_{k, j=1}^{3}\left(\frac{\partial \omega_{k}}{\partial x_{k}}-\frac{\partial \omega_{j}}{\partial x_{j}}\right)^{2}\right)+ \\
+\left(\beta-\frac{\eta^{2}}{\alpha}\right)(\operatorname{rot} \omega)^{2}+\alpha\left(\operatorname{rot} u+\frac{\eta}{\alpha} \operatorname{rot} \omega-2 \omega\right)^{2} .
\end{gathered}
$$

From (3) and (4), on the basis of the conditions satisfying the elastic constants, we can conclude that

$$
E\left(V^{\prime}, V\right)=E\left(V, V^{\prime}\right), \quad E(V, V) \geq 0 .
$$

Let now prove the following 
Theorem 1 Problems $(I)_{F, \varphi, \psi}$ and $(I I)_{F, \varphi, \psi}$ have no more than one solution.

Proof. We have to prove that the problems $(I)_{0,0,0}$ and $(I I)_{0,0,0}$ have only zero solutions. To this end, we apply formula (1) to the vectors $V=V(x, t)$ and $V^{\prime}=$ $\frac{\partial V(x, t)}{\partial t}$, where $V(x, t)=(u(x, t), \omega(x, t))$ is a regular solution of the problem $(I)_{0,0,0}$ or of the problem $(I I)_{0,0,0}$, and make use of the identities

$$
\begin{aligned}
V^{\prime} M V & =\frac{\partial V}{\partial t} M V=\left(\frac{\partial u}{\partial t}, \frac{\partial \omega}{\partial t}\right) \cdot\left(\rho \frac{\partial^{2} u}{\partial t^{2}}, \mathcal{J} \frac{\partial^{2} \omega}{\partial t^{2}}\right) \\
& =\frac{\rho}{2} \frac{\partial}{\partial t}\left|\frac{\partial u}{\partial t}\right|^{2}+\frac{\mathcal{J}}{2} \frac{\partial}{\partial t}\left|\frac{\partial \omega}{\partial t}\right|^{2} ; \\
E\left(V^{\prime}, V\right) & =E\left(\frac{\partial V}{\partial t}, V\right)=\frac{1}{2} \frac{\partial}{\partial t} E(V, V) .
\end{aligned}
$$

Then (1) takes the form

$$
\frac{\partial}{\partial t} \int_{D}\left(\frac{\rho}{2}\left|\frac{\partial u}{\partial t}\right|^{2}+\frac{\mathcal{J}}{2}\left|\frac{\partial \omega}{\partial t}\right|^{2}+\frac{1}{2} E(V, V)\right) d x=\int_{S} \frac{\partial V}{\partial t} T V d S .
$$

By virtue of the boundary conditions, the right-hand side of (5) for the both problems is equal to zero and, consequently,

$$
\int_{D}\left(\frac{\rho}{2}\left|\frac{\partial u}{\partial t}\right|^{2}+\frac{\mathcal{J}}{2}\left|\frac{\partial \omega}{\partial t}\right|^{2}+\frac{1}{2} E(V, V)\right) d x=\text { const } .
$$

Since this constant at the starting moment is equal to zero, it will remain unchanged in a due course, and $\left|\frac{\partial u}{\partial t}\right|^{2}=0,\left|\frac{\partial \omega}{\partial t}\right|^{2}=0 ; E(V, V)=0$ thus $V=(u, \omega) \equiv 0$.

\section{Green's Tensors and the Problems for Eigenval- ues}

As we will see below, in investigating the above-formulated dynamical problems, of great importance are the solutions of some special corresponding problems of statics which are called the Green's tensors.

The first Green's tensor or the Green's tensor of the first basic problem of statics corresponding to the problem $(I)_{F, \varphi, \psi}$ is called the matrix ${ }_{G}^{1}(x, y)$ of dimension $6 \times 6$, depending on two points $x$ and $y$ and satisfying the following conditions:

1) $\forall x, y \in D, x \neq y: \quad M(\partial x) \stackrel{1}{G}(x, y)=0$

2) $\forall z \in S, \forall y \in D: \stackrel{1}{G}(z, y)=0$

3) $\stackrel{1}{G}(x, y)=\Gamma(x-y)-\stackrel{1}{g}(x, y), x, y \in D$,

where $\Gamma(x-y)$ is the known matrix of fundamental solutions of the equation [3]

$$
M(\partial x) V(x)=0
$$


and $\stackrel{1}{g}(x, y)$ is a regular solution (including $x=y$ ) of equation (7) in the domain $D$.

It is clear that the proof of the existence of ${ }^{1}(x, y)$ is reduced to the solvability of the following first basic problem of statics for $D$ : find a regular in $D$ solution $\stackrel{1}{g}(x, y)$ of equation (7), satisfying the boundary condition

$$
\forall z \in S, \quad \forall y \in D: \stackrel{1}{g}(z, y)=\Gamma(z-y) .
$$

The solvability of such a problem has been proved in [3].

The second Green's tensor, or the Green's tensor of the second basic problem of statics in the domain $D$, corresponding to the problem $(I I)_{F, \varphi, \psi}$, cannot, generally speaking, be defined analogously to the first tensor. Since the second basic problem of statics is not always solvable [3], we, following to H. Weyl $[4,5]$, represent $\stackrel{2}{G}(x, y)$ in the domain $D$ in the form

$$
\stackrel{2}{G}(x, y)=\Pi(x, y)-\stackrel{2}{g}(x, y),
$$

where $\Pi(x, y)$ is the known matrix involving $\Gamma(x-y)$ and the vectors of rigid displacement, and $\stackrel{2}{g}(x, y)$ is a regular matrix in $D$, satisfying equation (7) and the boundary condition

$$
\forall z \in S, \quad \forall y \in D: \lim _{D \ni x \rightarrow z \in S} T(\partial x, n(x))^{2} g(x, y)=T(\partial z, n(z)) \Pi(z, y) .
$$

It is shown that the necessary and sufficient condition for the obtained second problem of statics is fulfilled and hence the existence of $\stackrel{2}{G}(x, y)$ is proved.

Relying on the results of $[3,6]$, we can show that the Green's tensors possess the following properties:

1) $\stackrel{k}{G}(x, y)=\stackrel{k}{G}{ }^{\top}(y, x)$, where " $\top$ " denotes transposition $k=1,2$;

2) $\forall(x, y) \in D \times D: \quad \stackrel{k}{G n}_{m n}(x, y)=O\left(|x-y|^{-1}\right)$,

$$
\frac{\partial}{\partial x_{j}} \stackrel{k}{G} m n(x, y)=O\left(|x-y|^{-2}\right)
$$

$$
k=1,2 ; \quad j=1,2,3 ; \quad m, n=\overline{1,6} ;
$$

3) $\forall(x, y) \in D \times D: \quad \frac{\partial}{\partial x_{j}} \stackrel{k}{G}(2)(x, y)=O(\ln |x-y|)$,

$$
j=1,2,3 ; \quad k=1,2 ; \quad m, n=\overline{1,6} ;
$$

4) $\forall(x, y) \in \bar{D}^{\prime} \times D: \frac{\partial^{2}}{\partial x_{i} \partial x_{j}} G_{m n}^{k}(x, y)=O\left(|x-y|^{-1}\right)$,

$$
k=1,2 ; \quad j=1,2,3 ; \quad m, n=\overline{1,6},
$$


where $|x-y|$ is the distance between the points $x$ and $y, \bar{D}^{\prime} \subset D$ is an arbitrary closed domain, lying strictly in $D$, and $G^{k}(2)(x, y)$ is an iterated kernel for $\stackrel{k}{G}(x, y)$ :

$$
\stackrel{k}{G}^{(2)}(x, y)=\int_{D} \stackrel{k}{G}(x, z) \stackrel{k}{G}(z, y) d z, \quad x \neq y, \quad k=1,2 .
$$

We rewrite equation (2) as follows:

$$
\widetilde{M}(\partial x) \widetilde{V}(x, t)-\frac{\partial^{2} \widetilde{V}(x, t)}{\partial t^{2}}=-\widetilde{F}(x, t)
$$

where $\widetilde{M}=\varkappa^{-1} M \varkappa^{-1}, \widetilde{V}=\varkappa V, \widetilde{F}=\varkappa^{-1} F, \varkappa=\left\|\sqrt{r_{k j}}\right\|_{6 \times 6}$.

Consider now two problems for eigen-values:

$$
\begin{array}{ll}
\forall x \in D: & \widetilde{M}(\partial x) W(x)+\gamma W(x)=0, \quad k=1,2 ; \\
\forall z \in S: & \lim _{D \ni x \rightarrow z \in S} \stackrel{1}{W}(x)=0, \quad \lim _{D \ni x \rightarrow z \in S} T(\partial x, n(x)) W(x)=0 .
\end{array}
$$

The first problem we denote by $(I)_{\gamma}$ and the second one by $(I I)_{\gamma}$. The eigen vector-function $\left.\stackrel{k}{W}(x)=\stackrel{k}{W}, \stackrel{k}{W}, \ldots, \stackrel{k}{W}, \ldots, W_{6}\right), k=1,2$ (not equal identically to zero) is said to be regular, if $\stackrel{k}{W}, \in C^{1}(\bar{D}) \cap C^{2}(D), i=\overline{1,6} ; k=1,2$.

It is not difficult to show [5] that the problems $(I)_{\gamma}$ and $(I I)_{\gamma}$ are equivalent to the following system of integral equations:

$$
\stackrel{k}{W}(x)=\gamma \int_{D} \stackrel{k}{K}(x, y) \stackrel{k}{W}(y) d y, \quad x \in D
$$

where $\stackrel{k}{K}(x, y)=\stackrel{k}{\varkappa} G(x, y) \varkappa, k=1,2$.

It follows from the above-mentioned properties of $\stackrel{k}{G}(x, y)$ that (11) is the integral equation with symmetric kernel of the class $L_{2}(D)$. Consequently, there exist a countable system of eigen-numbers $\left(\gamma_{n}\right)_{n=1}^{\infty},\left|\gamma_{n}\right| \rightarrow \infty$ as $n \rightarrow \infty$, and the corresponding orthonormalized in $D$ system of eigen-vectors $\left.\stackrel{k}{W^{n}}(x)\right)_{n=1}^{\infty}, x \in D, k=1,2$, of equation (11) or of the problems $(I)_{\gamma}$ and $(I I)_{\gamma}$, respectively. It is easy to state that all $\stackrel{1}{\gamma}_{n}>0$, while $\stackrel{2}{\gamma}_{n} \geq 0$, where $\stackrel{2}{\gamma}=0$ is the eigen sixth rank number, and the corresponding vectors are those of the rigid displacement $\left(\chi^{(n)}(x)\right)_{n=1}^{6}$. In what follows, it will be assumed that $\stackrel{2}{\gamma}_{n}=0, \stackrel{2}{W^{(n)}}=\chi^{(n)}, n=\overline{1,6}, \stackrel{2}{\gamma}_{n}>0$ for $n>6$. It can be shown that the vectors $\stackrel{k}{W}^{(n)}(x)$ are regular in the domain $D$. 
The Fourier method in three-dimensional dynamical ...

\section{Lemmas on the Order of Fourier Coefficients}

Lemma 1 For any six-component vector $\Phi(x)$ satisfying the conditions $\Phi \in C^{0}(\bar{D})$, $\frac{\partial \Phi}{\partial x_{i}} \in L_{2}(D), i=1,2,3 ;\left.\Phi\right|_{S}=0$ the inequality

$$
\sum_{n=1}^{\infty} \Phi_{n}^{2} \hat{\gamma}_{n} \leq \int_{D} E\left(\varkappa^{-1} \Phi, \varkappa^{-1} \Phi\right) d x
$$

where

$$
\Phi_{n}=\int_{D} \Phi(x) \stackrel{1}{W}^{(n)}(x) d x
$$

is valid. In particular, it follows from the above lemma that the numerical series in the left-hand side of (12) converges.

Proof. Applying Green's formula (11) to the vectors $V^{\prime}=\varkappa^{-1} \Phi(x)$ and $V=$ $\varkappa^{-1} W^{(n)}(x)$ and taking into account the condition $\left.\Phi\right|_{S}=0$, we obtain

$$
\int_{D} E\left(\varkappa^{-1} \Phi, \varkappa^{-1} W^{(n)}\right) d x=\gamma_{n}^{1} \Phi_{n} .
$$

In particular, assuming in (13) that $\Phi=\stackrel{1}{W}^{m}$, we obtain

$$
\int_{D} E\left(\varkappa^{-1} \underline{W}^{(m)}, \varkappa^{-1} \stackrel{1}{W}^{(n)}\right) d x=\left\{\begin{array}{lll}
1 & \text { for } & m=n \\
0 & \text { for } & m \neq n
\end{array}\right.
$$

Consider now a nonnegative value

$$
\mathcal{J}=\int_{D} E\left(\varkappa^{-1} V, \varkappa^{-1} V\right) d x
$$

and assume $V(x)=\Phi(x)-\sum_{n=1}^{n_{0}} \Phi_{n} \stackrel{1}{W}^{(n)}(x)$, then simple calculations show that

$$
\begin{gathered}
\mathcal{J}=\int_{\mathcal{J}} E\left(\varkappa^{-1} \Phi, \varkappa^{-1} \Phi\right) d x+\sum_{m, n=1}^{n_{0}} \int_{D} E\left(\varkappa^{-1}{ }^{1}{ }^{(m)}, \varkappa^{-1} W^{1}(n)\right) d x \\
-2 \sum_{n=1}^{n_{0}} \Phi_{n} \int_{D} E\left(\varkappa^{-1} \Phi, \varkappa^{-1} W^{1}(n)\right) d x .
\end{gathered}
$$

Taking into account (13) and (14), from (15) we find that

$$
\sum_{n=1}^{n_{0}} \Phi_{n}^{2} \stackrel{1}{\gamma}_{n} \leq \int_{D} E\left(\varkappa^{-1} \Phi, \varkappa^{-1} \Phi\right) d x
$$

which proves our lemma. 
Lemma 2 For any six-component vector $\Phi(x)$ satisfying the conditions $\Phi \in C^{0}(\bar{D})$, $\frac{\partial \Phi}{\partial x_{i}} \in L_{2}(D), i=1,2,3$, the inequality

$$
\sum_{n=1}^{\infty} \Phi_{n}^{2} \dot{\gamma}_{n}^{2} \leq \int_{D} E\left(\varkappa^{-1} \Phi, \varkappa^{-1} \Phi\right) d x
$$

where

$$
\Phi_{n}=\int_{D} \Phi(x) \stackrel{2}{W}^{(n)}(x) d x
$$

is valid.

Proof. Applying Green's formula to the vectors $V^{\prime}=\varkappa^{-1} \Phi(x)$ and $V=$ $\varkappa^{-1} \stackrel{2}{W}^{(n)}(x)$ and taking into account the condition $\left.T \stackrel{2}{W^{(n)}}\right|_{S}=0$, we obtain

$$
\int_{D} E\left(\varkappa^{-1} \Phi, \varkappa^{-1} \stackrel{2}{W}^{(n)}\right) d x=\stackrel{2}{\gamma}_{n} \Phi .
$$

Repeating further word for word the proof of Lemma 1, we will get inequality (16).

Lemma 3 For any six-component vector $\Phi(x)$ satisfying the conditions 1) $\Phi \in$ $\left.C^{1}(\bar{D}), 2\right) \frac{\partial^{2} \Phi}{\partial x_{i} \partial x_{j}} \in L_{2}(D)$ and 3$)\left.\Phi\right|_{S}=0$ for the problem $(I)_{F, \varphi, \psi}$ and the condition $\left.T \Phi\right|_{S}=0$ for the problem $(I I)_{F, \varphi, \psi}$, the inequality

$$
\sum_{n=1}^{\infty} \Phi_{n}^{2} \stackrel{k}{\gamma}^{2} \leq \int_{D}|\widetilde{M} \Phi|^{2} d x, \quad k=1,2
$$

is valid.

In particular, it follows from the above theorem that the numerical series in the left-hand side (17) converges.

Proof. Applying Green's formula (1) to the vectors $\varkappa^{-1} \Phi(x)$ and $\varkappa^{-1} W^{(n)}(x)$ and taking in each of the cases the boundary conditions $\Phi(x)$ and $\stackrel{k}{W^{(n)}}(x)$ on $S$, we obtain

$$
\int_{D} \widetilde{M} \Phi W^{k}(n) d x=-\stackrel{k}{\gamma}_{n} \Phi_{n}, \quad k=1,2,
$$

whence

$$
(\widetilde{M} \Phi)_{n}=-\stackrel{k}{\gamma} \Phi_{n}, \quad k=1,2 .
$$

For $\widetilde{M} \Phi(x)$, we write the Bessel's inequality

$$
\sum_{n=1}^{\infty}(\widetilde{M} \Phi)_{n}^{2} \leq \int_{D}|\widetilde{M} \Phi|^{2} d x
$$

Taking into account (18), from (19) we get (17). 
The Fourier method in three-dimensional dynamical ...

\section{Formal Scheme of the Fourier Method}

We denote $\widetilde{\varphi}(x)=\varkappa \varphi(x), \widetilde{\psi}(x)=\varkappa \psi(x)$. Let $\widetilde{V}^{I}(x, t)$ be a solution of the problem $(I)_{\widetilde{F}, \tilde{\varphi}, \widetilde{\psi}}$. We write the representation $\widetilde{V}^{I}(x, t)=\widetilde{V}^{1}(x, t)+\widetilde{V}^{2}(x, t)$, where $\widetilde{V}^{1}(x, t)$ is a solution of the problem $(I)_{0, \widetilde{\varphi}, \widetilde{\psi}}$, and $\widetilde{V}^{2}(x, t)$ is that of the problem $(I)_{\widetilde{F}, 0,0}$.

Applying to the problem $(I)_{0, \widetilde{\varphi}, \widetilde{\psi}}$ a formal scheme of the Fourier method, we obtain

$$
\widetilde{V}^{1}(x, y)=\sum_{n=1}^{\infty} W^{1}(n)(x)\left(\widetilde{\varphi}_{n} \cos \sqrt{{ }^{1}}{ }_{n} t+\frac{\widetilde{\psi}_{n}}{\sqrt{\gamma_{n}^{1}}} \sin \sqrt{\gamma_{n}} t\right)
$$

where

$$
\widetilde{\varphi}_{n}=\int_{D} \widetilde{\varphi}(x) \stackrel{1}{W}^{(n)}(x) d x, \quad \widetilde{\psi}_{n}=\int_{D} \widetilde{\psi}(x) \stackrel{1}{W}^{(n)}(x) d x .
$$

Formally, we decompose $\widetilde{V}^{2}(x, t)$ and $\widetilde{F}(x, t)$ into a series by the system $\left(\stackrel{1}{W}^{(n)}(x)\right)_{n=1}^{\infty}$ :

$$
\widetilde{V}^{2}(x, t)=\sum_{n=1}^{\infty} \widetilde{V}_{n}^{2}(t) \stackrel{1}{W}^{(n)}(x), \quad \widetilde{F}(x, t)=\sum_{n=1}^{\infty} \widetilde{F}_{n}(t) \stackrel{1}{W}^{(n)}(x),
$$

We get

$$
\widetilde{V}^{2}(x, t)=\sum_{n=1}^{\infty} W^{(n)}(x) \frac{1}{\sqrt{\frac{1}{\gamma_{n}}}} \int_{0}^{t} \widetilde{F}_{n}(\tau) \sin \sqrt{\gamma_{n}}(t-\tau) d \tau .
$$

Consequently, a solution of the problem $(I)_{\widetilde{F}, \widetilde{\varphi}, \widetilde{\psi}}$ is formally looks as follows:

$$
\begin{gathered}
\widetilde{V}^{I}(x, t)=\sum_{n=1}^{\infty} W^{(n)}(x)\left(\widetilde{\varphi}_{n} \cos \sqrt{\frac{1}{\gamma_{n}}} t+\frac{\widetilde{\psi}_{n}}{\sqrt{\frac{1}{\gamma_{n}}}} \sin \sqrt{\gamma_{n}} t\right) \\
+\sum_{n=1}^{\infty} W^{(n)}(x) \frac{1}{\sqrt{\frac{1}{\gamma_{n}}}} \int_{0}^{t} \widetilde{F}_{n}(\tau) \sin \sqrt{\frac{1}{\gamma_{n}}}(t-\tau) d \tau .
\end{gathered}
$$

Applying Green's formula (2) to the vectors $\varkappa^{-1} \widetilde{\varphi}(x)$ and $\varkappa^{-1} W^{(n)}(x)$ and taking into account that $\left.\widetilde{\varphi}\right|_{S}=\left.W^{(n)}\right|_{S}=0$, we obtain

$$
\int_{D} \widetilde{M} \widetilde{\varphi}(x) W^{(n)}(x)=-\gamma_{n}^{1} \widetilde{\varphi}_{n}
$$

that is,

$$
(\widetilde{M} \widetilde{\varphi})_{n}=-\stackrel{1}{\gamma}_{n} \widetilde{\varphi}_{n}
$$


We apply now formula (2) to the vectors $\varkappa^{-1} \widetilde{M} \widetilde{\varphi}(x)$ and $\varkappa^{-1} W^{(n)}(x)$ and take into account that $\left.\widetilde{M} \widetilde{\varphi}\right|_{S}=\left.\stackrel{1}{W}^{(n)}\right|_{S}=0$. Thus we get

$$
\left(\widetilde{M}^{2} \widetilde{\varphi}\right)_{n}=-\stackrel{1}{\gamma}_{n}(\widetilde{M} \widetilde{\varphi})_{n}
$$

whence by virtue of (21), we obtain

$$
\widetilde{\varphi}_{n}=\frac{\left(\widetilde{M}^{2} \widetilde{\varphi}\right)_{n}}{\gamma_{n}^{2}}
$$

Analogously, we find that

$$
\widetilde{\psi}_{n}=-\frac{(\widetilde{M} \widetilde{\psi})_{n}}{1_{n}}, \quad \widetilde{F}_{n}(t)=-\frac{(\widetilde{M} \widetilde{F})_{n}(t)}{\stackrel{1}{\gamma}_{n}} .
$$

In view of (22) and (23), (20) takes the form

$$
\begin{gathered}
\widetilde{V}^{I}(x, t)=\sum_{n=1}^{\infty} \frac{\underline{W}^{(n)}(x)}{\stackrel{1}{\gamma}_{n}^{2}}\left(\widetilde{M^{2}} \widetilde{\varphi}\right)_{n} \cos \sqrt{\gamma_{n}} t \\
-\sum_{n=1}^{\infty} \frac{\underline{W}^{(n)}(x)}{\stackrel{1}{\gamma}_{n}^{3 / 2}(\widetilde{M} \widetilde{\varphi})_{n} \sin \sqrt{\hat{\gamma}_{n}} t} \\
-\sum_{n=1}^{\infty} \frac{\stackrel{1}{W}^{(n)}}{\frac{1}{\gamma}_{n}^{3 / 2}} \int_{0}^{t}(\widetilde{M} \widetilde{F})_{n}(\tau) \sin \sqrt{\gamma_{n}}(t-\tau) d \tau
\end{gathered}
$$

Let $\widetilde{V}^{I I}(x, t)$ be a solution of the problem $(I I)_{\widetilde{F}, \widetilde{\varphi}, \widetilde{\varphi}}$. Then, analogously, we get

$$
\begin{gathered}
\widetilde{V}^{I I}(x, t)=\sum_{n=1}^{6} \chi^{(n)}(x)\left(\widetilde{\varphi}_{n}+t \widetilde{\psi}_{n}\right)+\sum_{n=7}^{\infty} \frac{\stackrel{2}{W}^{(n)}(x)}{\stackrel{2}{\gamma}_{n}^{2}}\left(\widetilde{M}^{2} \widetilde{\varphi}\right)_{n} \cos \sqrt{\stackrel{2}{\gamma}_{n}} t \\
-\sum_{n=7}^{\infty} \frac{\stackrel{2}{W}^{(n)}(x)}{\stackrel{2}{\gamma}_{n}^{3 / 2}}(\widetilde{M} \widetilde{\psi})_{n} \sin \sqrt{\stackrel{2}{\gamma}_{n}} t+\sum_{n=1}^{6} \chi^{(n)}(x) \int_{0}^{t}\left(\int_{0}^{t} \widetilde{F}_{n}(\tau) d \tau\right) d t \\
-\sum_{n=7}^{\infty} \frac{\stackrel{2}{W}^{(n)}(x)}{\stackrel{2}{\gamma}_{n}^{3 / 2}} \int_{0}^{t}(\widetilde{M} \widetilde{F})_{n}(\tau) \sin \sqrt{\gamma_{n}^{2}}(t-\tau) d \tau
\end{gathered}
$$

\section{Justification of the Fourier Method}

To justify the Fourier method, we have to prove that the series appearing in (24) and (25) and those obtained by means of a single termwise differentiation of these 
series converge uniformly in the closed cylinder $\bar{\Omega}$, while the series obtained by a double termwise differentiation of these series converge uniformly in the cylinder $\Omega$.

We investigate here only the series appearing in (24) because those appearing in (25) are investigated analogously. First, we investigate the series

$$
\sum_{n=1}^{\infty} \frac{W^{(n)}(x)}{\gamma_{n}^{2}}\left(\widetilde{M}^{2} \widetilde{\varphi}\right)_{n} \cos \sqrt{\gamma_{n}} t
$$

where for the sake of simplicity we adopt $\stackrel{1}{W}^{(n)}=W^{(n)}$ and $\stackrel{1}{\gamma}=\gamma_{n}$.

Simultaneously, we investigate the series obtained by a single and double termwise differentiation with respect to $t$ of the series (26),

$$
\begin{aligned}
& -\sum_{n=1}^{\infty} \frac{W^{(n)}(x)}{\gamma_{n}^{3 / 2}}\left(\widetilde{M}^{2} \widetilde{\varphi}\right)_{n} \sin \sqrt{\gamma_{n}} t \\
& \sum_{n=1}^{\infty} \frac{W^{(n)}(x)}{\gamma_{n}}\left(\widetilde{M}^{2} \widetilde{\varphi}\right)_{n} \cos \sqrt{\gamma_{n}} t
\end{aligned}
$$

Estimate a residual of the series (26) by using the Cauchy-Bunyakowski's inequality and majorizing simultaneously the cosine by unity. We have

$$
\left|\sum_{n=m}^{m+p} \frac{W^{(n)}(x)}{\gamma_{n}^{2}}\left(\widetilde{M}^{2} \widetilde{\varphi}\right)_{n} \cos \sqrt{\gamma_{n}} t\right| \leq\left[\sum_{n=m}^{m+p} \frac{\left|W^{(n)}(x)\right|^{2}}{\gamma_{n}^{4}} \sum_{n=m}^{m+p}\left(\widetilde{M}^{2} \widetilde{\varphi}\right)_{n}^{2}\right]^{1 / 2} .
$$

Since $\widetilde{M}^{2} \widetilde{\varphi} \in L_{2}(D)$, by virtue of Bessel's inequality we obtain

$$
\sum_{n=1}^{\infty}\left(\widetilde{M}^{2} \widetilde{\varphi}\right)_{n}^{2} \leq \int_{D}\left|\widetilde{M}^{2} \widetilde{\varphi}\right|^{2} d x
$$

In view of (30), it follows from (29) that to prove that the series (26) converges uniformly in $\bar{\Omega}$, it suffices to state that the sum of the series

$$
\sum_{n=1}^{\infty} \frac{\left|W^{(n)}(x)\right|^{2}}{\gamma_{n}^{4}}
$$

exists and is uniformly bounded in $\bar{D}$.

The Bessel's inequality provides us with

$$
\sum_{n=1}^{\infty} \frac{\left|W^{(n)}(x)\right|^{2}}{\gamma_{n}^{2}} \leq \int_{D}|K(x, y)|^{2} d y
$$

where $K(x, y)=\stackrel{1}{K}(x, y)=\stackrel{1}{\leftarrow}(x, y) \varkappa$. By virtue of (8), it follows from (32) that the sum of the series

$$
\sum_{n=1}^{\infty} \frac{\left|W^{(n)}(x)\right|^{2}}{\gamma_{n}^{2}}
$$


exists and is uniformly bounded in $\bar{D}$.

The same conclusion is especially valid for the series (31). The series (27) and (28) are investigated analogously.

Consider now the series obtained by a single and double termwise differentiation with respect to $x$ of the series (26),

$$
\begin{aligned}
& \sum_{n=1}^{\infty} \frac{\frac{\partial W^{(n)}(x)}{\partial x_{i}}}{\gamma_{n}^{2}}\left(\widetilde{M}^{2} \widetilde{\varphi}\right)_{n} \cos \sqrt{\gamma_{n}} t, \quad i=1,2,3 \\
& \sum_{n=1}^{\infty} \frac{\frac{\partial^{2} W^{(n)}(x)}{\partial x_{i} \partial x_{j}}}{\gamma_{n}^{2}}\left(\widetilde{M}^{2} \widetilde{\varphi}\right)_{n} \cos \sqrt{\gamma_{n}} t, \quad i=1,2,3
\end{aligned}
$$

Consider the iterated kernel $K^{(2)}(x, y)$ for the kernel $K(x, y)$. Then

$$
W^{(n)}(x)=\gamma_{n}^{2} \int_{D} K^{(2)}(x, y) W^{(n)}(y) d y .
$$

The Bessel's inequality results in

$$
\begin{gathered}
\sum_{n=1}^{\infty} \frac{\left|\frac{\partial W^{(n)}(x)}{\partial x_{i}}\right|^{2}}{\gamma_{n}^{4}} \leq \int_{D}\left|\frac{\partial K^{(2)}(x, y)}{\partial x_{i}}\right|^{2} d y, \quad i=1,2,3 \\
\sum_{n=1}^{\infty} \frac{\left|\frac{\partial^{2} W^{(n)}(x)}{\partial x_{i} \partial x_{j}}\right|^{2}}{\gamma_{n}^{4}} \leq \int_{D}\left|\frac{\partial^{2} K^{(2)}(x, y)}{\partial x_{i} \partial x_{j}}\right|^{2} d y, \quad i, j=1,2,3 .
\end{gathered}
$$

By virtue of (9), it follows from (36) that the sum of the series appearing in the left-hand side of (36) exists and is uniformly bounded in $\bar{D}$.

On the strength of (10), it follows from (37) that the sum of the series appearing in the left-hand side of (37) exists and is uniformly bounded in $\bar{D}^{\prime}$, where $\bar{D}^{\prime} \subset D$ is an arbitrary closed domain lying strictly in $D$.

Repeating the above reasoning, we can prove that the series (34)

converges uniformly in $\bar{\Omega}$, and the series (35) converges uniformly in $\Omega$. form

Let us now pass to the investigation of the second series (24). We rewrite it in the

$$
\sum_{n=1}^{\infty} \frac{W^{(n)}(x)}{\gamma_{n}^{2}}\left((\widetilde{M} \widetilde{\psi})_{n} \sqrt{\gamma_{n}}\right) \sin \sqrt{\gamma_{n}} t .
$$

Comparing the series (38) and (26), it is not difficult to notice that they are of the same structure, the only difference is that the cosine is replaced by the sine and $\left(\widetilde{M}^{2} \widetilde{\varphi}\right)_{n}$ is replaced by $(\widetilde{M} \widetilde{\varphi})_{n} \sqrt{\gamma_{n}}$. In investigating the series $(26)$ we have used the fact that the series

$$
\sum_{n=1}^{\infty}\left(\widetilde{M}^{2} \widetilde{\varphi}\right)_{n}^{2}
$$


converges. As for the convergence of the series

$$
\sum_{n=1}^{\infty}(\widetilde{M} \widetilde{\psi})_{n}^{2} \gamma_{n}
$$

it follows directly from the above-proven Lemma 1. Consequently, we can apply the above scheme to the series (38).

Finally, we investigate the third series appearing in (24) and rewrite it in the form

$$
\sum_{n=1}^{\infty} \frac{W^{(n)}(x)}{\gamma_{n}^{2}} \int_{0}^{t}(\widetilde{M} \widetilde{F})_{n}(\tau) \sqrt{\gamma_{n}} \sin \sqrt{\gamma_{n}}(t-\tau) d \tau .
$$

It is clear from the above reasoning that we have to prove the convergence of the series

$$
\sum_{n=1}^{\infty} \int_{0}^{t}\left((\widetilde{M} \widetilde{F})_{n}(\tau)\right)^{2} \gamma_{n} d \tau
$$

The last statement follows directly from Lemma 1 and the well-known theorem on the limiting passage under the Lebesgue integral sign.

It remains for us to prove that the Fourier series

$$
\sum_{n=1}^{\infty} \widetilde{F}_{n}(t) W^{(n)}(x)
$$

of the vector-function $\widetilde{F}(x, t)$ converges uniformly in the closed cylinder $\bar{\Omega}$. Consider the series

$$
\sum_{n=1}^{\infty} W^{(n)}(x) \int_{0}^{t} \frac{d \widetilde{F}_{n}(\tau)}{d \tau} d \tau
$$

and estimate the residual of the series (43) by means of the Cauchy-Bunyakowski's inequality

$$
\begin{gathered}
\left|\sum_{n=m}^{m+p} W^{(n)}(x) \int_{0}^{t} \frac{d \widetilde{F}_{n}(\tau)}{d \tau} d \tau\right| \\
\leq\left[\sum_{n=m}^{m+p} \frac{\left|W^{(n)}(x)\right|^{2}}{\gamma_{n}^{2}} \sum_{n=m}^{m+p} \int_{0}^{e}\left|\frac{d \widetilde{F}_{n}(\tau)}{d \tau}\right|^{2} \gamma_{n}^{2} d \tau\right]^{1 / 2}
\end{gathered}
$$

Using Lemma 3 for the vector-function $\frac{\partial \widetilde{F}(x, t)}{\partial t}$ and the theorem on the limiting passage under the integral sign, we can state that the series

$$
\sum_{n=1}^{\infty} \int_{0}^{e}\left|\frac{d \widetilde{F}_{n}(\tau)}{d \tau}\right|^{2} \gamma_{n}^{2} d \tau
$$

converges. 
Thus taking into account the fact that the sum of the series (31) is uniformly bounded, it follows from (7) that the series (41) converges uniformly in $\bar{\Omega}$.

From (41), we have

$$
\sum_{n=1}^{\infty} W^{(n)}(x) \int_{0}^{t} \frac{d \widetilde{F}_{n}(\tau)}{d \tau} d \tau=\sum_{n=1}^{\infty} \widetilde{F}_{n}(t) W^{(n)}(x)-\sum_{n=1}^{\infty} \widetilde{F}_{n}(0) W^{(n)}(x) .
$$

Thus it is clear from (43) that to prove that the series (40) converges uniformly in $\bar{\Omega}$, it suffices to prove that the series

$$
\sum_{n=1}^{\infty} \widetilde{F}_{n}(0) W^{(n)}(x)
$$

converges uniformly in $\bar{D}$. We estimate the residual of the series (44),

$$
\left|\sum_{n=m}^{m+p} \widetilde{F}_{n}(0) W^{(n)}(x)\right| \leq\left[\sum_{n=m}^{m+p} \frac{\left|W^{(n)}(x)\right|^{2}}{\gamma_{n}^{2}} \sum_{n=m}^{m+p} \widetilde{F}_{n}{ }^{2}(0) \gamma_{n}^{2}\right]^{1 / 2} \text {. }
$$

Using Lemma 3 for $\widetilde{F}(x, 0)$, we immediately find that the series

$$
\sum_{n=1}^{\infty} \widetilde{F}_{n}^{2}(0) \gamma_{n}^{2}
$$

converges uniformly. Taking now into account that the sum of the series (31) is uniformly bounded, we can conclude from (45) that the series (44) converges uniformly in $\bar{D}$. Consequently, a full justification of the Fourier method for the problems under consideration is complete.

Thus we have proved the following

Theorem 2 If $F, \varphi$ and $\psi$ are the given vector-functions satisfying the conditions mentioned in item 2 , then the series (24) and (25) are the regular (classical) solutions of the problems $(I)_{F, \varphi, \psi}$ and $(I I)_{F, \varphi, \psi}$, respectively.

\section{References}

[1] E. L. Aéro and E. V. Kuvshinskii, Continuum theory of asymmetric elasticity. The problem of "internal" rotation. (Russian) Fiz. Tverd. Tela 5 2591-2598 (Russian); translated as Soviet Physics Solid State 51963 1892-1897.

[2] J. P. Nowacki and W. Nowacki, Some problems of hemitropic micropolar continuum. Bull. Acad. Polon. Sci. Sér. Sci. Tech. 25 (1977), no. 4, 297-305.

[3] L. Giorgashvili, Solution of fundamental boundary value problems of statics for a hemitropic micropolar medium. (Russian) Tbiliss. Gos. Univ. Inst. Prikl. Mat. Trudy 16 (1985), 56-80. 
[4] H. Weyl, Das asymptotische Verteilungsgesetze der Eigenschwingungen eines beliebig gestalteten elastischen Korpers. Rend. Circ. Mat. Palermo 39(1915), 1-49.

[5] V. D. Kupradze, T. G. Gegelia, M. O. Basheleishvili and T. V. Burchuladze, Three-dimensional problems of the mathematical theory of elasticity and thermoelasticity. Translated from the second Russian edition. Edited by V. D. Kupradze. North-Holland Series in Applied Mathematics and Mechanics, 25. North-Holland Publishing Co., Amsterdam-New York, 1979.

[6] D. Natroshvili, Estimates of Green's tensors of elasticity theory and their applications. (Russian). Tbilis. Univ., Tbilisi, 1978.

DOI: $10.7862 /$ rf.2012.1

Yuri Bezhuashvili

email: iuri1946@mail.ru

Roland Rukhadze

Department of Mathematics,

Georgian Technical University,

77, M. Kostava Str.,

Tbilisi 0175, Georgia

Received 21.10.2011, Revisted 6.12.2011 